\title{
Shape correction of thin mirrors in a reconfigurable modular space telescope
}

\author{
Keith Patterson, Sergio Pellegrino and James Breckinridge \\ Graduate Aerospace Laboratories, California Institute of Technology, \\ 1200 E. California Blvd, Pasadena, CA 91125
}

\begin{abstract}
In order to facilitate the construction of future large space telescopes, the development of low cost, low mass mirrors is necessary. However, such mirrors suffer from a lack of structural stability, stiffness, and shape accuracy. Active materials and actuators can be used to alleviate this deficiency. For observations in the visible wavelengths, the mirror surface must be controlled to an accuracy on the order of tens of nanometers. This paper presents an exploration of several mirror design concepts and compares their effectiveness at providing accurate shape control. The comparison test is the adjustment of a generic mirror from its manufactured spherical shape to the shape required by various off-axis mirrors in a segmented primary mirror array. A study of thermal effects is also presented and, from these results, a recommended design is chosen.
\end{abstract}

Keywords: thin mirrors, segmented telescopes, shape correction, image quality

\section{INTRODUCTION}

Space-based telescopes have been constrained by the size of available launch vehicles. The James Webb Space Telescope, currently under construction, will be the first space observatory with a folding primary mirror. This is a major step forward in space telescope technology, however the cost and complexity associated with folding mirror architectures and the size limitations still inherent in this approach will ultimately necessitate even more radically different approaches. Considering the time it takes for a new space observatory concept to reach maturity, the search for such alternatives should begin now.

We have recently participated in a study of large space apertures sponsored by the Keck Institute of Space Studies (KISS). ${ }^{1}$ This study put forward the concept of forming a large mosaic mirror through on-orbit self-assembly of relatively small mirror segments mounted on separate low-cost spacecraft, building on related developments at MIT. $^{2,3}$ These satellites would be able to execute autonomous docking maneuvers and latch together to form a single coherent mirror surface. ${ }^{1}$ As a follow-on to this study, we have been studying the feasibility of a low-cost technology for lightweight mirrors to be used on each of the spacecraft. The mirrors that we are considering have a thickness on the order of millimeters, diameter up to $1 \mathrm{~m}$ and could be manufactured on a single mandrel. Active shape correction is used to achieve the required shape accuracy. In order to reduce the launch mass, the mirror substrate's thickness and backing structure should be minimized and also the actuation scheme should be internally reacted; hence surface parallel actuation will be adopted. Various patterns of active materials on the back surface of a thin mirror substrate will be studied and compared. The proposed approach could conceivably be extended to larger diameter thin mirrors that could be rolled up, along the lines suggested by Romeo et al. ${ }^{4}$

A key advantage of the proposed approach is that, unlike existing primary mirror architectures, where the stiffness of the mirror structure is such that the shape of the mirror can be changed only by small amounts, in the present scheme the use of a flexible structure provides a much wider range of shape adaptability. This property opens up new possibilities which will be demonstrated by showing that the mirror segments can even be moved around to reconfigure the shape of the aperture.

Further author information:

Keith Patterson: kdpatter@caltech.edu

Sergio Pellegrino: sergiop@caltech.edu

James Breckinridge: jbreckin@caltech.edu

Space Telescopes and Instrumentation 2010: Optical, Infrared, and Millimeter Wave, edited by Jacobus M. Oschmann Jr., Mark C. Clampin, Howard A. MacEwen, Proc. of SPIE Vol. 7731, 773121 - @ 2010 SPIE · CCC code: 0277-786X/10/\$18 · doi: 10.1117/12.861442

Proc. of SPIE Vol. $7731773121-1$ 
This paper examines the performance of various mirror actuation schemes in a segmented mirror architecture and in the presence of significant thermal loads. Clearly, the stiffness, thickness, and coefficient of thermal expansion (CTE) of the mirror and its active materials will all play significant roles in the optical performance of the telescope. Instead of relying on thermal insulation and/or sunshades to keep the temperature of the mirrors relatively constant, we explore the feasibility of using materials with near-zero coefficient of thermal expansion and to use the actuation layer to correct for both shape errors and thermal distortions.

The paper is organized as follows: The next section presents a brief review of recent developments in thin mirror technologies and also reviews the mirror performance metrics that will be used in the paper. Section 3 formulates the shape correction problem and presents the approach utilized to determine the best shape achievable when given a set of actuators. Section 4 proposes a nanosatellite mission concept to demonstrate key aspects of a reconfigurable space telescope that is autonomously assembled in orbit and operates at visible wavelengths. A study of the mirror shape corrections required for this mission is presented in Section 5 , including materials with near-zero thermal expansion. Section 6 concludes the paper.

\section{BACKGROUND}

This section presents a brief review of recent advances in thin mirrors and defines the performance metrics that will be used to compare different designs.

\subsection{Thin mirror designs}

There have been several recent efforts to produce thin mirrors for space telescopes, some of which will be briefly reviewed here.

The creation of doubly-curved thin films has been achieved with processes aimed at minimizing surface roughnesses, thickness variation, and thermal expansion coefficients. ${ }^{5}$ Mirror substrates were formed by casting polyimide or other polymers on high-quality mandrels and then depositing a reflective coating of aluminum on one side of the substrate. A variety of different structural configurations have been considered, including pressurized inflatables, edge-tensioned flat surfaces, or freestanding shell structures.

One method of controlling the surface figure is to provide a number of external actuators along the boundary edge of a thin shell mirror (see Figure 1). By moving the outer edge of the mirror one has a certain control authority over the entire mirror surface, but this method has difficulty in achieving high shape accuracies simultaneously near the edge of the mirror and in the inner region.

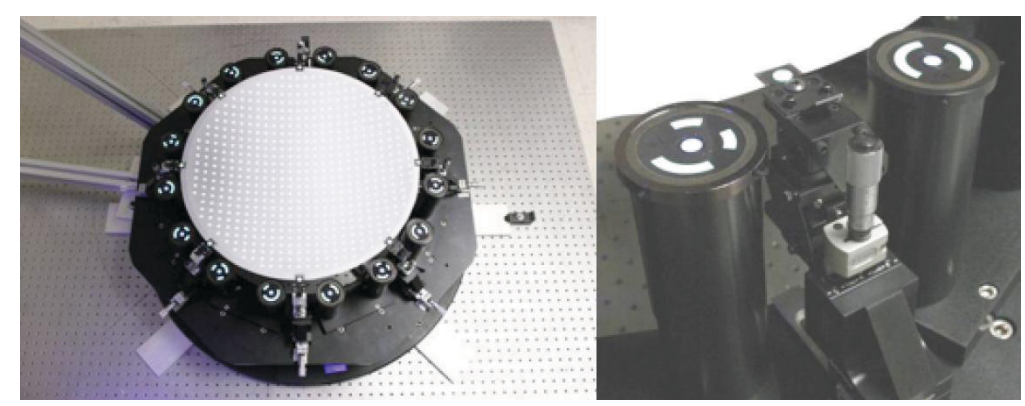

Figure 1. Thin mirror controlled by boundary actuation ${ }^{12}$

There have also been investigations into using active materials bonded to a thin mirror substrate to provide internally reacted actuation across the entire mirror surface. An illustration of this type of surface parallel actuation is shown in Figure 2. Hickey et al. ${ }^{6}$ have demonstrated nanolaminate thin shell spherical mirrors manufactured via chemical deposition onto a shaped mandrel. The nanolaminate layers have thicknesses on the order of $100 \mu \mathrm{m}$ and are bonded to a stiff $\mathrm{SiC}$ backing structure that is fitted with actuating elements. The complete mirror structure has an areal density of around $9.5 \mathrm{~kg} / \mathrm{m}^{2}$ including actuators.

Demonstrations of surface parallel actuation on thin mirror substrates have been shown. ${ }^{7,8}$ This design involves a flat silicon substrate with a layer of deposited PZT patches as seen in Figure 3. By varying the voltages 


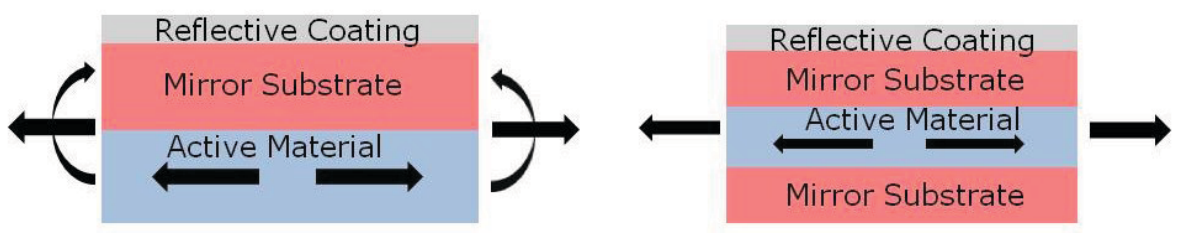

Figure 2. Examples of surface parallel actuation

applied to the different PZT patches, various Zernike mode deformations were demonstrated. PZT has good actuation characteristics but is very brittle and hence great care is required to ensure mechanical integrity of the actuating patches. Another possible actuator material is the piezoelectric polymer, PVDF. Figure 4 shows a slack polyimide membrane attached to a stiff edge structure; a continuous layer of PVDF has been cast on the backside of the polyimide substrate. By actuating the PVDF layer the mirror becomes taut. An advantage of using PVDF instead of PZT or other piezoelectric and electrostrictive-type materials was found by the author to be its flexibility and durability.

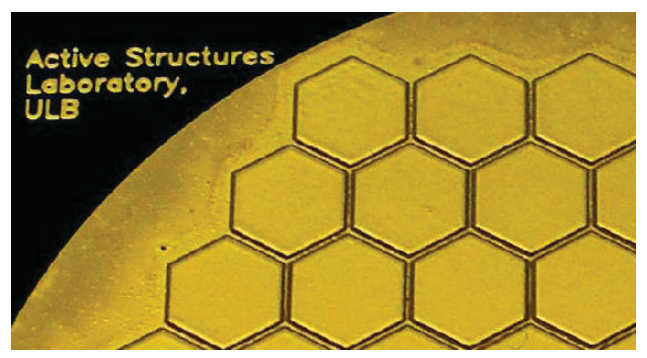

Figure 3. Silicon bimorph mirror with deposited, hexagonal PZT patches. ${ }^{7}$

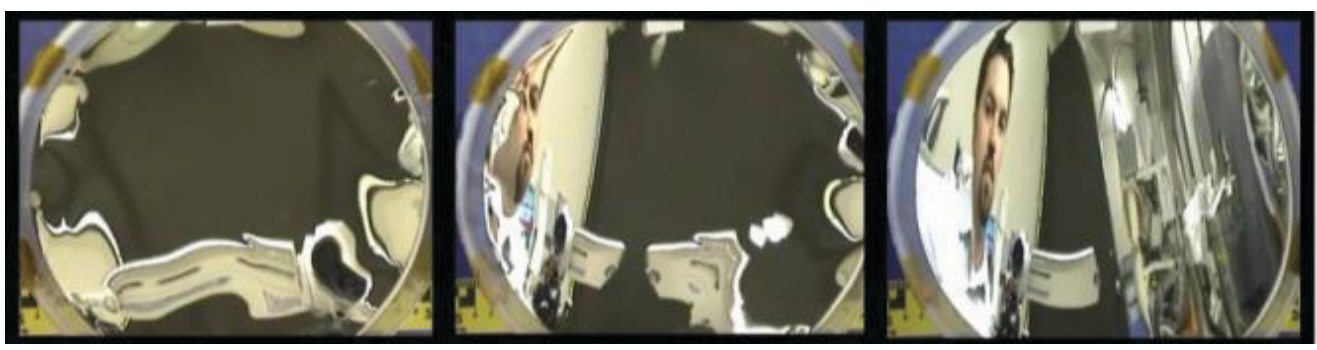

Figure 4. Thin mirror bonded to PVDF actuating layer. ${ }^{8}$

\subsection{Mirror performance metrics}

\subsubsection{Point spread function}

The Point Spread Function (PSF) of an optical system is the image that the system provides for a point source of light (e.g. a distant star) in object space. Due to diffraction, the point source will not map to a single point in the image plane, and the light intensity will be spread out over the image. The PSF for a perfect, circular aperture is the Airy pattern, as shown in Figure 5. The width of the central peak, which defines how spread out the light is over the image, is governed by the diameter of the aperture. Larger diameter apertures result in narrower peaks. Other features of the PSF such as the rate of attenuation from the center, and regions of extremely low intensity, may also be important depending on the application. The actual aperture configuration (segmented, sparse, etc.), alignment and surface error of the mirrors, etc. will all affect the PSF. Of particular relevance to the present study, the PSF becomes more smeared as the mirror surface error grows. An extended object can be modeled as a collection of point sources of light, and the convolution of the object with the system PSF closely represents the system's output image. If the PSF is very spread out, then this will result in loss of contrast and blurring in the image. If one is trying to resolve two objects that have a very small angular spacing, then it is important to achieve and maintain a narrow PSF during image exposure. 


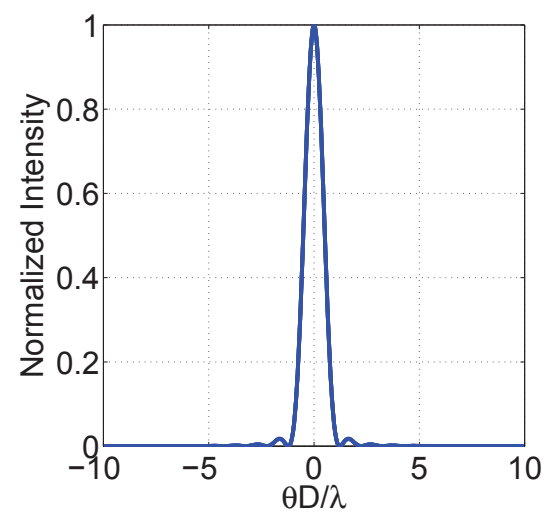

(a)

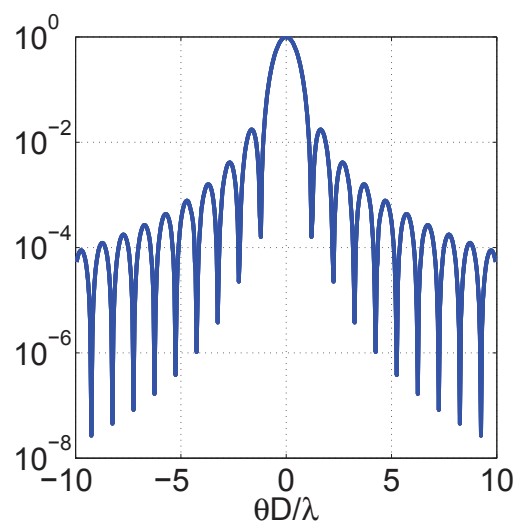

(b)

Figure 5. Normalized PSF intensity for a perfect circular aperture shown on: (a) linear scale and (b) logarithmic scale. $\theta$ is the angular coordinate in the image, $D$ the aperture diameter and $\lambda$ the observed wavelength.

\subsubsection{RMS surface error}

The RMS surface error error is a simple scalar measure of shape-related performance. It is particularly convenient for comparison purposes and it will be utilized as an initial criterion for mirror design. The RMS surface error is not a substitute for the PSF and the modulation transfer function (MTF) (discussed in the following section) as a merit function to verify performance of the mirror within the system.

To maintain a PSF of good quality the RMS surface error of the mirror should be minimized. ${ }^{9}$ It should be noted that in a real mirror there are several different factors contributoring to the RMS error. The high spatial frequency component of the RMS error will be governed by the mirror surface roughness; this is an issue related to manufacturing techniques and processes that bears little relation with shape correction. On the other hand, minimization of the low and mid frequency components of the RMS error may be achieved through the use of sufficient numbers of actuators to bend or stretch the mirror into the desired shape. Further discussion of the modeling and minimization of surface error RMS is found in Section 3.1.

\subsubsection{Modulation transfer function}

The two-dimensional Fourier transform of the PSF is the optical transfer function (OTF) and its magnitude is the modulation transfer function (MTF). An image of the MTF is a representation of how well features of the target object or scene with various spatial frequencies will be preserved through the telescope into an image. An example MTF is shown in Figure 6, along with some dimensions that can be used as scalar representations of the MTF. The center region of the image is for low spatial frequency information, and regions towards the edges of the image are for high spatial frequency content. For further information, Fiete et al. ${ }^{10}$ provide an excellent introduction to and discussion of the MTF.

\section{APPROACH AND METHODOLOGY}

The general problem that will be addressed is as follows: Given a thin mirror that, for various reasons (e.g. design, manufacture, thermal distortion) has the wrong shape for the required application, is it possible to correct the shape of the mirror by means of a set of surface-parallel actuators? A successful correction is one that reduces the RMS surface error to less than a specified threshold.

This is an optimization problem that could be approached at different levels. In the present numerical study we have considered several different arrangements of the actuators and for each arrangement we have determined the density of independent actuating elements for which the required degree of correction is achieved when the best possible actuation efforts are applied. The best possible efforts are defined as those that minimize the RMS surface error and their values are determined as follows: 


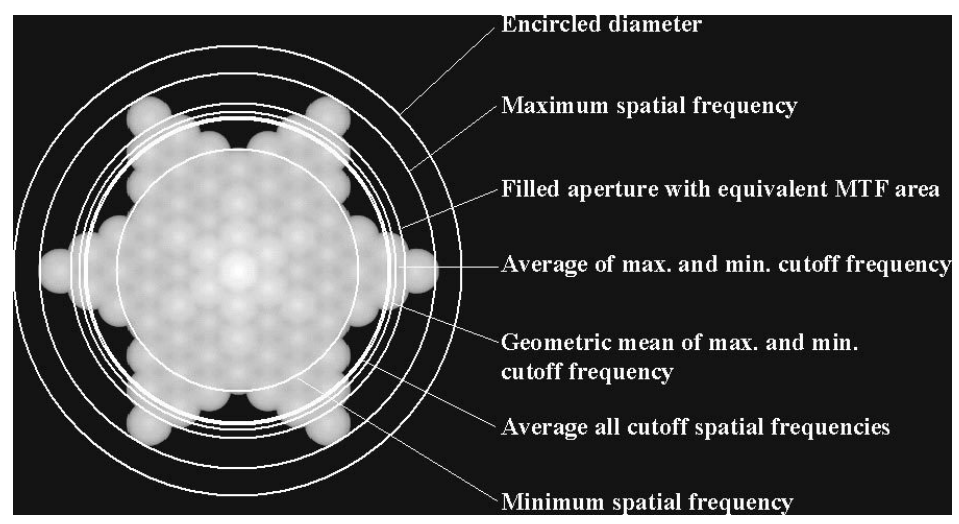

Figure 6. Example MTF showing different possible scalar measures of performance. ${ }^{10}$

1. Geometry generation (Matlab)

2. Finite element model (Abaqus)

3. Find influence functions (Python script)

4. Assemble problem and solve (Matlab)

5. Post-process data (Matlab)

The mirror and actuator geometries are generated in Matlab based on the desired parameters. The geometry is fed into the finite element software Abaqus/Standard ver. 6.8 using its Python scripting interface, and the influence function for each actuator is obtained from the elastic deformation of the mirror. Once all of the influence functions are determined, then the shape fitting problem is solved in Matlab, along with extra visualization and post-processing.

\subsection{Shape correction}

The numerical techniques for mirror surface control are well established and will be reviewed here. Suppose that there exists a mirror surface mesh with $m$ number of nodes, and there is an associated control system with $n$ actuators. Now suppose that associated with the $i^{\text {th }}$ actuator is a column vector, $\mathbf{a}_{i} \in \Re^{m}, i=1 . . n$, that is linearly independent from the other vectors, corresponding to the other actuators. This column vector is formed by finding the nodal deflections of the finite element model due to a unit input (e.g. 1 volt) to the associated actuator, and turning off all other actuators. This column vector is known as the influence vector, since it determines the influence that the actuator has on the mirror surface. Example influence functions are shown in Section 4.1 in Figure 10. The influence vectors are assembled into an influence matrix, A:

$$
\mathbf{A}=\left[\begin{array}{llll}
\mathbf{a}_{1} & \mathbf{a}_{2} & \ldots & \mathbf{a}_{n}
\end{array}\right] \in \Re^{m \times n}
$$

The assumption is made that all deviations from the initial surface shape are small. This is easily justified by noting that most surface shape adjustments are on the order of visible wavelengths (hundreds of nanometers), whereas the characteristic mirror dimensions are on the order of centimeters or meters. This assumption allows linear combinations of the influence vectors to be used to predict the mirror deflections. Hence, the influence matrix can be used to transform a control vector, $\mathbf{u} \in \Re^{n}$, consisting of the actuator input values, into a shape deflection vector, $\delta \in \Re^{m}$, which contains the deflection of all nodal points of the mirror mesh. Thus, the control vector and shape deflection vector are related via the influence matrix, as follows:

$$
\mathrm{Au}=\delta
$$

In general, a mirror will start with a certain shape, $\mathbf{s}_{1} \in \Re^{m}$, and the desired shape change is $\mathbf{s}_{2} \in \Re^{m}$. Then, the desired deflection vector is $\boldsymbol{\delta}=\mathbf{s}_{2}-\mathbf{s}_{1}$. Even assuming that the appropriate vector norm (e.g. 2-norm or RMS)of $\boldsymbol{\delta}$ is small, this deflection vector will, in general, not belong to the rangespace of $\mathbf{A}$. Therefore, the appropriate control vector would come from a least squares (LS) solution of (2). 
For a general mirror mesh, it is desirable to weight each of the nodal deflections by an appropriate associated surface area, $S_{i}$, such that the fitting solution is made independent of the mesh structure. In this study, an approximation for these areas is found by projecting the mirror surface nodes down onto a plane, computing the Voronoi area ${ }^{11}$ for each node, and then projecting the areas back onto the mirror surface via the direction cosine of the surface normal. These area weights are arranged along the diagonal of a matrix, $\mathbf{W} \in \Re^{m \times m}$. (2) is then modified as follows:

$$
\mathrm{WAu}=\mathrm{W} \delta
$$

One possible approach to find the weighted LS solution is through reduced QR factorization of the matrix product WA. A brief overview of the method is as follows:

$$
\begin{gathered}
\mathbf{W A u}=\widehat{\mathbf{Q}} \widehat{\mathbf{R}} \mathbf{u}=\mathbf{W} \boldsymbol{\delta} \\
\widehat{\mathbf{R}} \mathbf{u}=\widehat{\mathbf{Q}}^{\mathrm{T}} \mathbf{W} \boldsymbol{\delta}
\end{gathered}
$$

Once the right hand side of (5) is computed, then due to the fact that $\widehat{\mathbf{R}}$ is upper triangular, a simple backsubsitution method is employed to compute $\mathbf{u}$. Then, re-application of $\mathbf{A}$ results in an approximation to the original $\boldsymbol{\delta}$. The difference between the approximation and the original is the residual vector or residual shape error, $\mathbf{r}=\mathbf{A} \mathbf{u}-\boldsymbol{\delta} \in \Re^{m}$. Accounting for the weights in the residual, $\hat{\mathbf{r}}=\mathbf{W A u}-\mathbf{W} \boldsymbol{\delta} \in \Re^{m}$. For convenience, the weights in $\mathbf{W}$ are now changed to be the square roots of $S_{i}$ non-dimensionalized by the total mirror surface area. Thus, the 2-norm of $\hat{\mathbf{r}}$ is now equivalent to the RMS surface error with units of length:

$$
\|\hat{\mathbf{r}}\|_{2}=\sqrt{\hat{\mathbf{r}}^{\mathrm{T}} \hat{\mathbf{r}}}=\sqrt{(\mathbf{A u}-\boldsymbol{\delta})^{\mathrm{T}} \mathbf{W}^{2}(\mathbf{A u}-\boldsymbol{\delta})} \equiv \sqrt{\frac{\sum_{i} r_{i}^{2} S_{i}}{\sum_{i} S_{i}}}
$$

\section{PROPOSED DEMONSTRATION MISSION}

A technology demonstration of key parts of the proposed reconfigurable telescope concept is envisioned. The plan is to mount the primary mirror segments on independent spacecraft to allow for assembly and disassembly capabilities. These spacecraft would be able to undock, re-arrange themselves into a new configuration, and then re-dock (see Figure 7). To allow for imaging operations in the different configurations, the mirror segments must be able to vary their shapes. Note that high quality imaging is not among the objectives of this small-scale demonstration mission, as a much larger number of mirrors will be required for this.

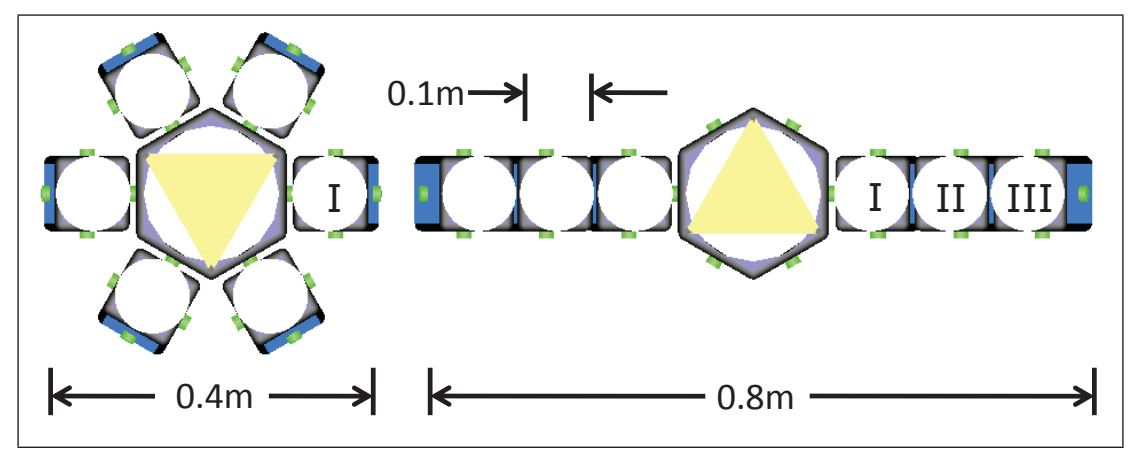

Figure 7. Spacecraft configurations: compact and wide apertures.

\subsection{Problem definition}

It is assumed that each of the mirror segments is manufactured as a $0.1 \mathrm{~mm}$ thick polyimide spherical shell with radius of curvature of $2 \mathrm{~m}$. The actuating material is also $0.1 \mathrm{~mm}$ thick and is bonded to the back side of the shell. Assumed material properties are found in Table 1. A concept for the individual mirrors is shown in Figure 8. By having all segments start out identical, a single mandrel can be used for the manufacturing, thus 


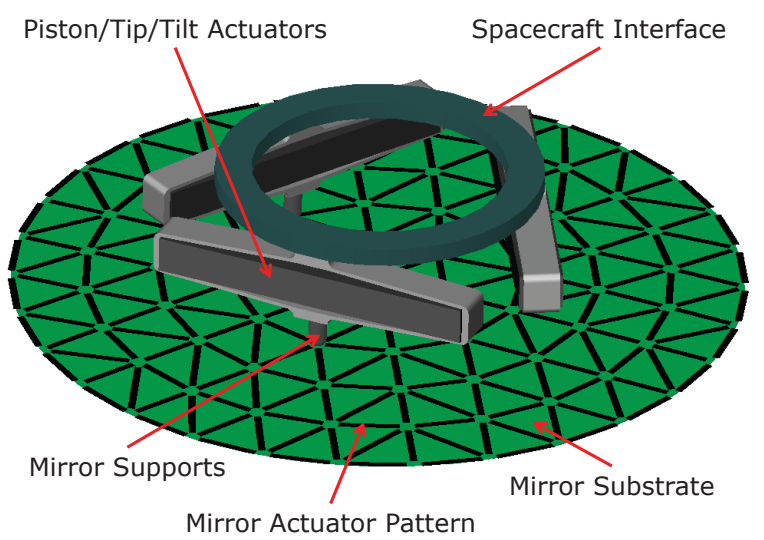

Figure 8. Back view of mirror concept with example actuator pattern and rigid body supports. The three example piston/tip/tilt actuators shown are Cedrat ${ }^{\circledR}$ APA $^{\text {TM }}$ piezoelectric actuators.

reducing costs. Also, the segments are all interchangeable which increases the overall reliability of the system. However, the mirrors would then require rigid body positioning adjustments on the order of a millimeter, as well as shape correction. The three unique positions to be studied are labeled in Figure 7. The remaining unlabeled positions are simply rotations of the labeled ones.

Table 1. Assumed representative material properties.

\begin{tabular}{|c||c|c|c|c|}
\hline & $\begin{array}{c}\text { Elastic Modulus } \\
(\mathrm{MPa})\end{array}$ & Poisson's Ratio & $\begin{array}{c}\text { Thermal Expansion } \\
\left(\times 10^{-6} /{ }^{\circ} \mathrm{C}\right)\end{array}$ & $\begin{array}{c}\text { Piezoelectric Coefficient } \\
\mathrm{d}_{31}\left(\times 10^{-12} \mathrm{~V} / \mathrm{m}\right)\end{array}$ \\
\hline \hline Mirror Substrate & 3000 & 0.34 & 20 & - \\
\hline Active Material & 3000 & 0.34 & 30 & 23 \\
\hline
\end{tabular}

The ideal shape of the segmented primary mirror will be assumed to be a paraboloid with a $1 \mathrm{~m}$ focal length, and it will be the task of the actuators to change each spherical mirror into an off-axis segment of a paraboloidal surface. The wavelength of interest is $\lambda=633 \mathrm{~nm}$ and hence all results will be shown as percentages of $\lambda$. Typically an RMS error of $\lambda / 10$ or $\lambda / 20$ is acceptable.

The initial shape errors (with piston, tip, and tilt removed) for the three positions are shown in Figure 9. For simplicity, the initial, spherical curvature was chosen to be equal to that at the center of the paraboloid. This choice, which may not be optimal, leads to a growth of the shape errors for segments further away from the axis of the paraboloid. The piston and tilt components of the error have already been removed from the plots under the assumption that the rigid body mirror positioning will be accounted for by the three out-of-plane actuators. Correction of these shape errors will be the test for comparing different patterns of actuators.

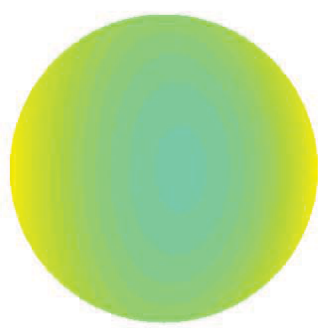

(a)

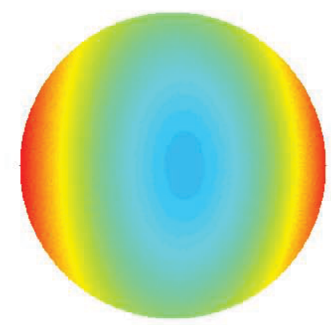

(b)

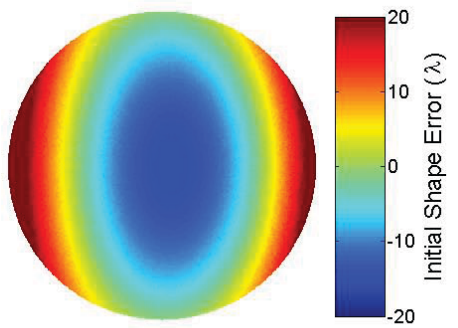

(c)

Figure 9. Initial shape error (with piston/tip/tilt removed) for the positions: (a) I, (b) II, and (c) III. 
Four different patterns of active material were studied to determine which provided the best shape correction. The patterns are shown in Figure 10. The filled triangular and hexagonal patches have low directionality in their influence functions. The bar actuators have a greater degree of directionality, but the characteristic length scales of those patterns are necessarily larger in order to maintain roughly equal numbers of actuators between the filled patch and bar patterns, for fair comparison purposes. All patterns are regular and even though the mirror boundary is circular, at this point of the study the actuator patterns are simply truncated near the edges.

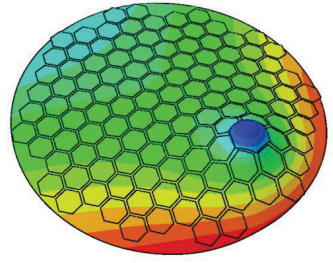

(a)

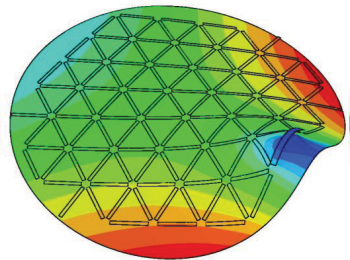

(b)

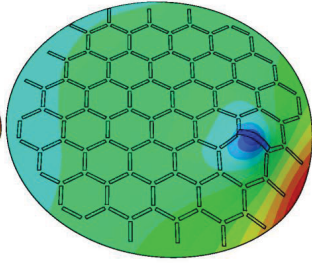

(c)

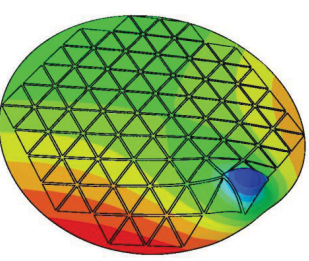

(d)

Figure 10. Four actuator patterns with approximately the same number of actuators showing exaggerated example influence functions: (a) hexagonal patches, (b) triangular bars, (c) hexagonal bars, (d) triangular patches.

\section{RESULTS}

\subsection{Shape control}

After generating the influence matrices for each actuator pattern and solving for the best fit to a $1 \mathrm{~m}$ focal length paraboloid, the residual errors for the triangular bar and hexagonal bar patterns were found to be about an order of magnitude smaller than those of the hexagonal patch and triangular patch patterns. The required actuator efforts and resulting error residuals for a mirror that goes to Position III (the worst case position) of the mirror array can be seen in Figure 11. Whereas the bar patterns had a fairly consistent residual pattern towards the mirror edge, the patch patterns suffered from large residual peaks and valleys near the edges, although the error was fairly uniform for a small region in the mirror center. The residual patterns for the other mirror positions were very similar but with smaller amplitudes and are therefore not shown for brevity. A summary of the actuator efforts and RMS values are reported in Table 2. Note that the actuator voltage values are reported assuming that the material is PVDF with constant piezoelectric constant of $23 \times 10^{-12} \mathrm{~m} / \mathrm{V}$.

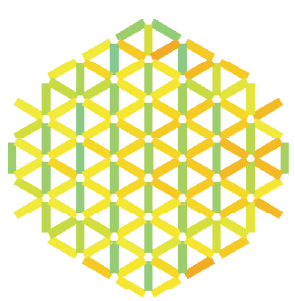

(a)

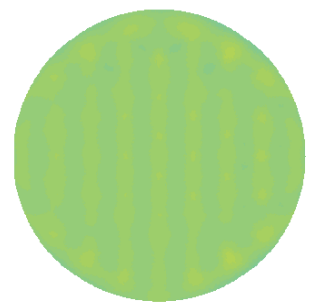

(e)

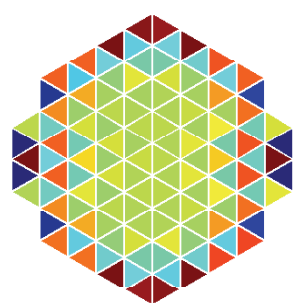

(b)

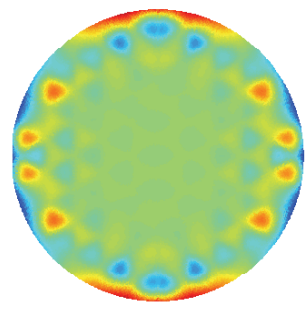

$(\mathrm{f})$

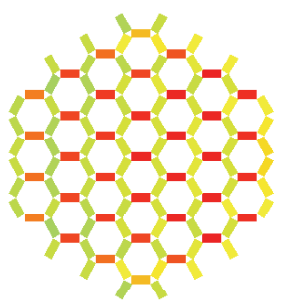

(c)

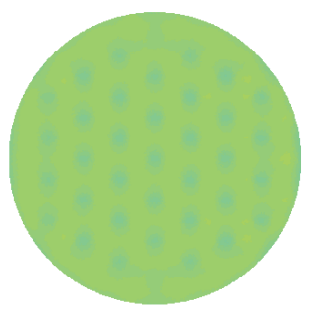

(g)

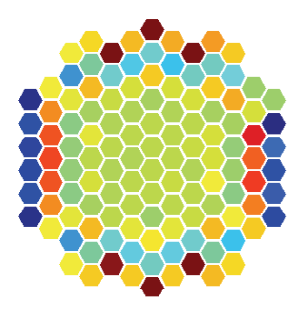

(d)

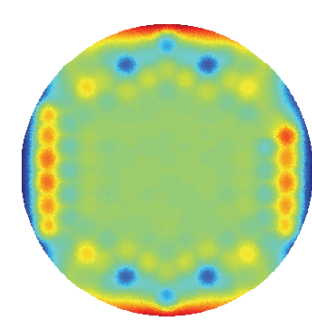

(h)
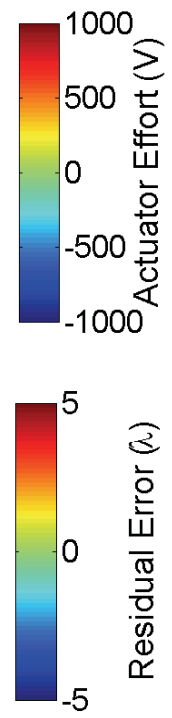

3
$\frac{3}{2}$
$\frac{1}{\pi}$
$\frac{0}{0}$
$\frac{0}{0}$
$\frac{0}{\alpha}$

Figure 11. Pattern residuals for Position III: (a) - (d) show the actuator pattern and required actuator voltages. (e) - (h) display the corresponding residual errors. Some values from this figure may be found in the following Table 2 . 
Table 2. RMS residual error (fraction of $\lambda$ ) of four patterns and total voltage range (V) in brackets.

\begin{tabular}{|c||c|c|c|c|}
\hline & Triangular Bars & Triangular Patches & Hexagonal Bars & Hexagonal Patches \\
\hline \hline Position I & $0.0174(95.7)$ & $0.1427(394.6)$ & $0.0197(147.2)$ & $0.1667(330.6)$ \\
\hline Position II & $0.0475(251.3)$ & $0.4293(1,156.5)$ & $0.0575(387.1)$ & $0.5072(330.6)$ \\
\hline Position III & $0.0922(473.8)$ & $0.8525(2,272.9)$ & $0.1134(735.6)$ & $1.0070(2,021.2)$ \\
\hline
\end{tabular}

\subsection{Further investigation}

The materials assumed for the first part of the study (see Table 1) would not be suitable for use in an environment that is not thermally controlled because they have large CTE values. They were used as a starting point for the study of active material patterns. Our collaborators in the KISS study are developing a bimetallic film with near-zero CTE (see the photo in Figure 12). It is believed that through a combination of this novel material, or alternative low CTE plastics, and an active material layer (e.g. PVDF or PZT), a thin mirror with the required characteristics could be realized. Also, while PVDF would be desirable as the active material due to its toughness, it remains to be seen whether the required actuation strains will be achievable. If not, then deposited patterns of PZT, with its much higher piezoelectric coefficient, will likely become necessary.
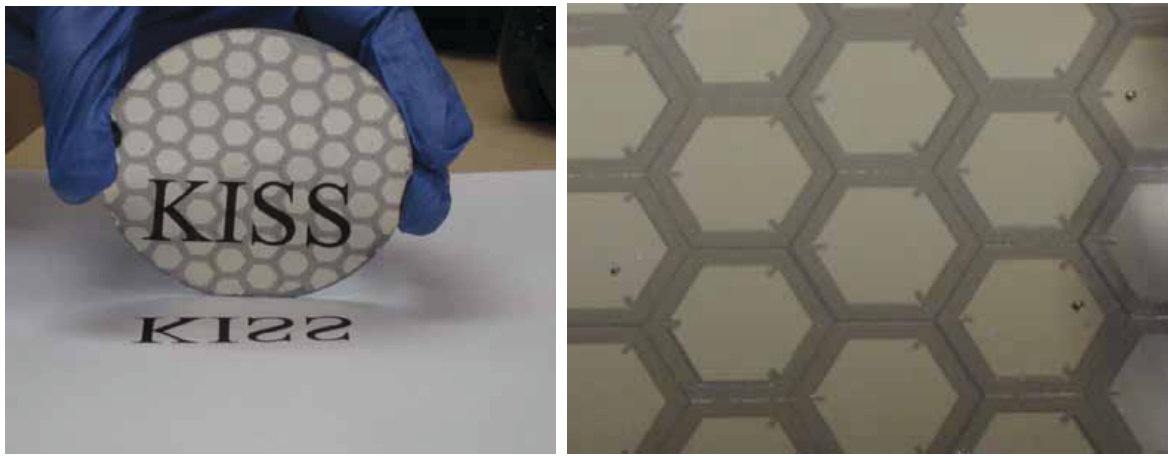

Figure 12. Early fabricated bimetallic mirror film with large scale pattern made by a deposition process (courtesy of Prof. Daraio and Dr Misra).

The triangular bar pattern was chosen for further investigation due to the fact that it had the smallest shape errors and because of the lower actuator voltages that it requires. The hexagonal patch pattern was also carried along as a comparison, and because of its potential for good thermal performance. The first additional study was into the effect of a bulk temperature change on the mirror/actuator assembly. The regularity of the triangular pattern causes it to not reach the edge of the circular mirror, therefore, it turned out to have significant residual thermal distortion near the edge. For this reason, the triangular pattern was modified as shown in Figure 13 to better accommodate a circular shape. Partial patches were also added to the hexagonal patch pattern in order to improve its edge control.

Figure 14 displays the dependence of the residual RMS error after shape correction for each array position, along with two thermal deformation cases, on the number of actuators in each pattern. The horizontal, black dashed line indicates the desired $\lambda / 10$ residual. This figure confirms the better performance of the triangular bar pattern over the hexagonal patch pattern. The thermal error curves show the effect of a bulk temperature change of $100^{\circ} \mathrm{C}$ and subsequent correction, for each pattern. The uppermost (teal) curve shows the residual error in the case of a polyimide substrate with large CTE. As might be expected, the thermal residual error is much larger than the initial shape residual error. A more stable substrate has been considered for comparison. In the limit, if the mirror substrate CTE were to be exactly zero, then the thermal residual error would also be exactly zero. The mirror itself would not change shape and the actuators would compensate their own thermal strains with equal and opposite piezoelectric strains. The purple curve shows the thermal error when the mirror substrate is replaced by a polyimide with a CTE of $0.4 \mathrm{ppm} /{ }^{\circ} \mathrm{C}$. This value is based on NeXolve's Novastrat ${ }^{\mathrm{TM}} 300$ polyimide 


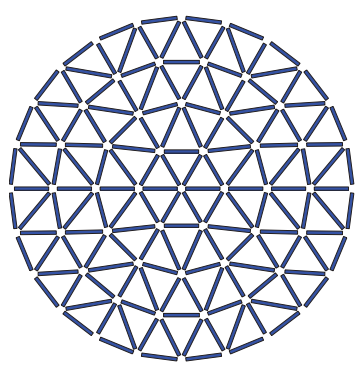

(a)

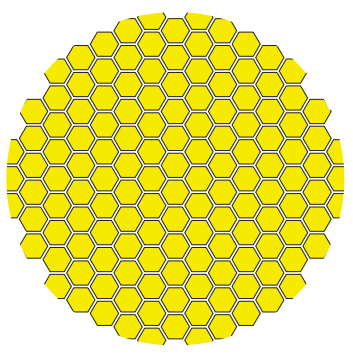

(b)

Figure 13. (a) Triangular pattern mapped out to a circle and (b) partial patches added to the hexagonal pattern.

material, which maintains this value over a very large temperature range. ${ }^{8}$ In this case, the thermal residual error falls in the same range of the initial shape correction errors, and so at sufficiently high actuator densities, the error recedes into the desired range. A triangular bar pattern with approximately 240 actuators would appear to be sufficient to keep the initial shape correction and thermal residuals below the $\lambda / 10$ limit. For this case, the maximum strain induced in the mirror film is just below $50 \times 10^{-6}$ (see Figure 15).

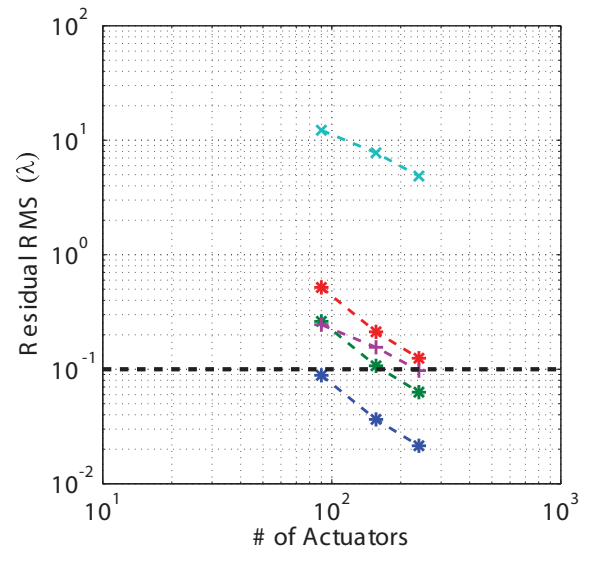

(a)

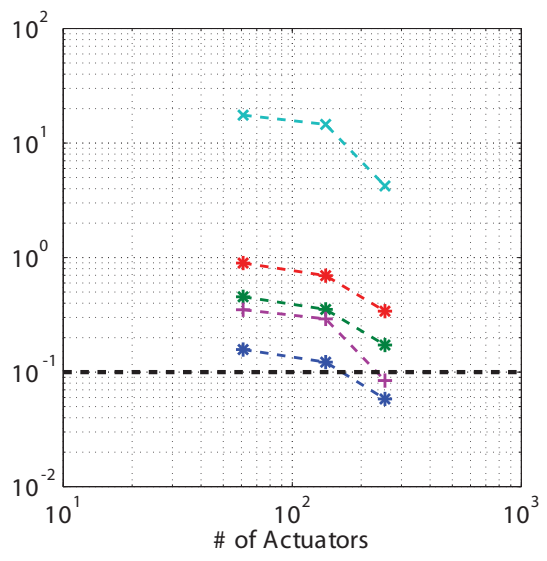

(b)

Figure 14. Residual error of (a) mapped triangular bar pattern and (b) hexagonal patch pattern for five cases: initial shape correction in three positions and bulk temperature change of $100^{\circ} \mathrm{C}$ with two different CTE values.

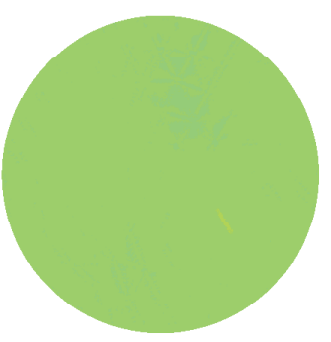

(a)

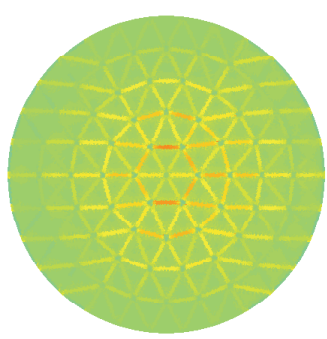

(b)

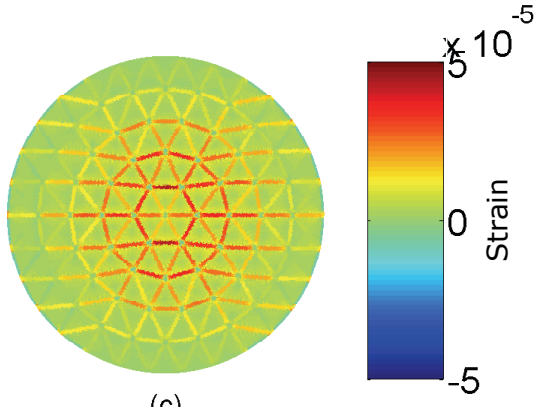

(c)

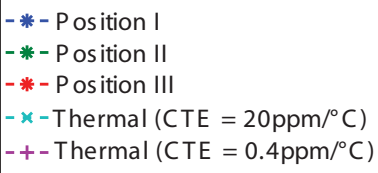

Figure 15. Maximum principal strains (unitless) in the mirror substrate after initial shape correction with 240 mapped triangular bar actuators. (a) Position I (b) Position II (c) Position III 


\subsection{Effects on system PSF and MTF}

The wavefront error (WFE) introduced into the telescope by the primary mirror segments is double the mirror segments' residual shape error, due to reflection. The wavefront error normalized by the observation wavelength can be transformed into a phase shift using the complex exponential and multiplied by the amplitude (reflectance) of the aperture to produce the pupil function. Neglecting other effects, such as misalignments of the secondary mirror and detector, its 2D spatial Fourier transform provides a simple diffraction model of the system PSF. In the case of 240 actuators arranged in a mapped triangular pattern with initial shape residual errors only, i.e. thermally induced shape errors are not considered, the PSF's and MTF's in Figures 16 and 17 are obtained for the two configurations of the telescope. The reference PSF and MTF for the given apertures with no wavefront phase error are also shown for comparison. The degradation in the PSF and MTF is more prominent in the wide configuration of the telescope, as expected, due to the larger errors in Positions II and III.

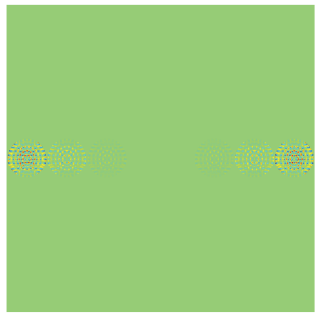

(a)

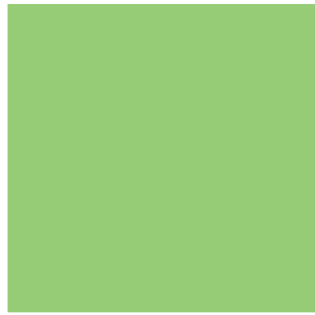

(e)

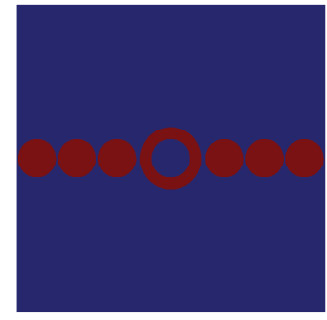

(b)

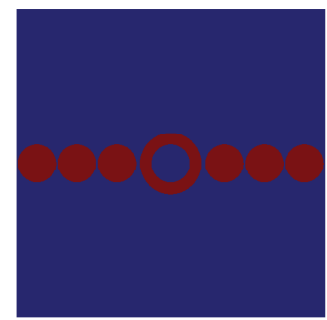

(f)

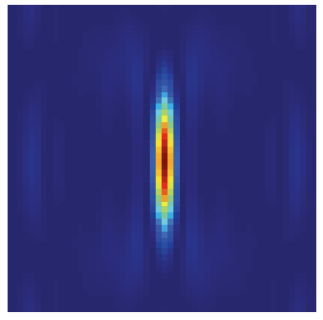

(c)

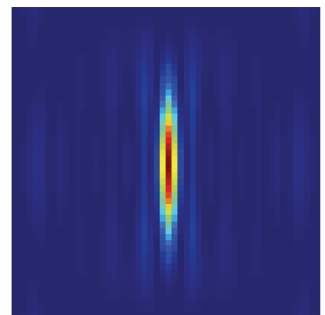

(g)

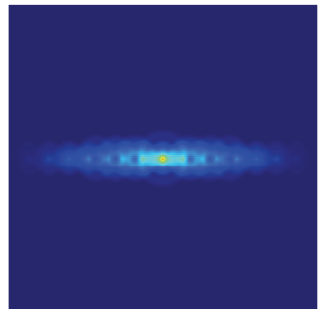

(d)

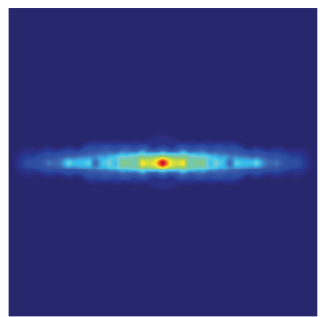

(h)

Figure 16. Wide configuration of telescope using 240 actuators per mirror segment in mapped triangular pattern; (a) WFE from initial shape correction residual, (b) wide aperture and (c) resulting PSF and (d) MTF. (e) - (h) PSF and MTF for wide aperture with zero WFE as a reference comparison for results in top row.

\section{CONCLUSION}

As a result of the modeling work presented in this paper, the proposed mirror design uses the mapped triangular bar pattern with over 240 actuators for each mirror segment. This number of actuators should be sufficient to maintain good shape control of the primary mirror. The mirror substrate should have a low CTE. Future work will involve the fabrication of mirror prototypes using polyimide or bimetallic films and bonded to PVDF or PZT actuators. Material testing and subsequent mirror performance validation will enable detailed mirror design work. Effort must be given into the design of the wavefront sensing and mirror actuator control systems. If work on these demonstration mirrors proves fruitful, then this study will be extended towards the design of large mirror segments (1 meter diameter) in the hope of enabling new telescope architectures.

\section{ACKNOWLEDGMENTS}

This research was sponsored by the Keck Institute for Space Studies (KISS). We would like to thank Dr Mark Lake and Dr Matt Mountain for stimulating comments during the initial formulation of the concepts presented in this paper. Professor Chiara Daraio and Dr Abha Misra have kindly allowed us to present some early results from their research on low CTE, bimetallic mirror films. The nanosatellite mission proposed in Section 4 is a collaboration between Caltech and the University of Surrey. We acknowledge the contribution of Dr Craig Underwood (University of Surrey, UK) to the formulation of this mission concept. 


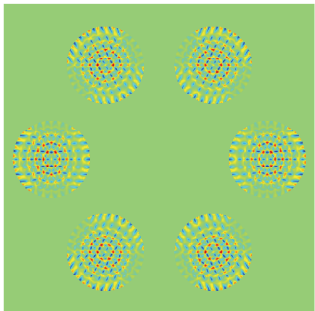

(a)

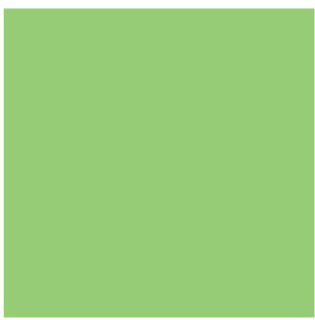

(e)

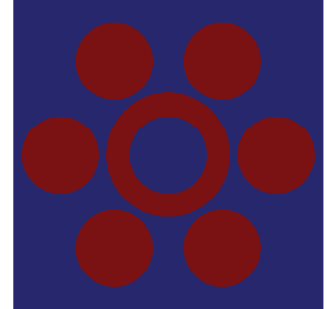

(b)

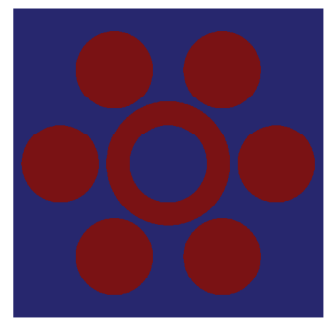

(f)

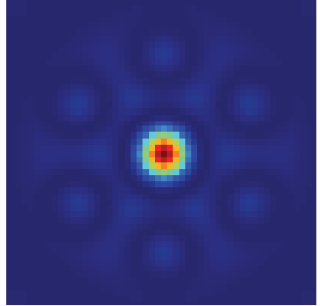

(c)

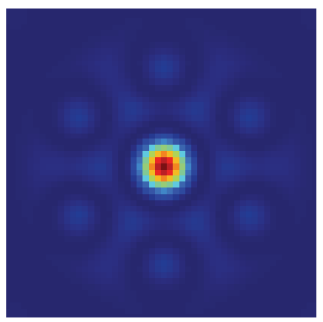

(g)

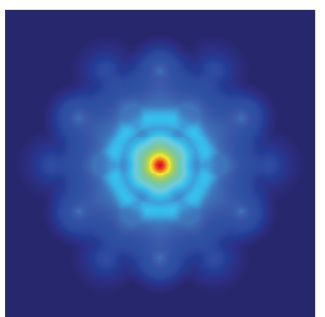

(d)

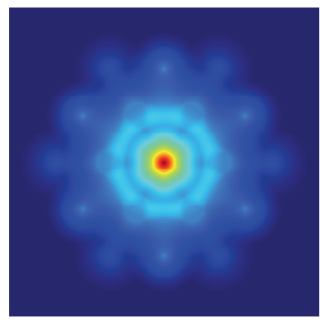

(h)

Figure 17. Same as Fig. 16 but for compact configuration.

\section{REFERENCES}

[1] Breckinridge, J.B., Dooley, J., Ortiz, M. and Pellegrino, S., Large Space Apertures (LSA) Study Report, Keck Institute of Space Studies (2009).

[2] Katz, J.G. "Estimation and Control of Flexible Space Structures for Autonomous On-Orbit Assembly, M.Sc. thesis in Aeronautics and Astronautics at MIT (2009).

[3] Rodgers, L.P. "Concepts and Technology Development for the Autonomous Assembly and Reconfiguration of Modular Space Systems," M.Sc. thesis in Mechanical Engineering (2005).

[4] Romeo, R.C., Meinel, A.B., Meinel, M.P. and Chen, P.C., "Ultra-lightweight and hyper-thin rollable primary mirror for space telescopes," in UV, Optical, and IR Space Telescopes and Instruments, Breckinridge, J.B., ed., vol. 4013. SPIE, pp. 634-639 (2000).

[5] Blonk, B., Moore, J., Patrick, B., and Flint, E., "Membrane mirrors in space telescopes," in Recent Advances in Gossamer Spacecraft, Jenkins, C., ed., 45-108, American Institute of Aeronautics and Astronautics, Inc., Reston, Virginia (2006).

[6] Hickey, G., Barbee, T., Ealey M., and Redding, D. "Actuated Hybrid Mirrors for Space Telescopes," SPIE Astronomical Telescopes and Instrumentation 7731-71 (2010).

[7] Rodrigues, G., Bastaits, R., Roose, S., Stockman, Y., Gebhardt, S., Schoenecker, A., Villon, P., and Preumont, A., "Modular bimorph mirrors for adaptive optics," in [Optical Engineering], Volume 48, Issue 3, pp. 034001-034001-7 (2009).

[8] Pearson, J., Moore, J., and Fang, H., "Large and high precision inflatable membrane reflector," 51st AIAA/ASME/ASCE/AHS/ASC Structures, Structural Dynamics, and Materials Conference, AIAA, Orlando, Florida (2010).

[9] P. Bely, The Design and Construction of Large Optical Telescopes, Springer, New York (2003).

[10] Fiete, R., Tantalo, T., Calus, J., and Mooney, J., "Image quality of sparse-aperture designs for remote sensing," in [Optical Engineering], Volume 41, pp. 1957-1969 (2008).

[11] R. Klein, Concrete and Abstract Voronoi Diagrams, Springer-Verlag, Berlin (1987).

[12] Lindler, J. and Flint, E., "Robustness of thin film shells with discrete boundary actuation," 47th AIAA/ASME/ASCE/AHS/ASC Structures, Structural Dynamics, and Materials Conference (2006). 